\title{
Successful use of Single dose of Methotrexate for the treatment of Unruptured Ectopic Pregnancy at Tribhuvan University Teaching Hospital, Kathmandu, Nepal
}

\author{
Rawal S
}

Department of Obs/Gyn, TU Teaching Hospital, Kathmandu, Nepal

\begin{abstract}
Ectopic pregnancy continues to be one of the most common gynecologic emergencies. The incidence of ectopic pregnancies which are diagnosed much earlier with the help of advanced tools is in the increasing trend, probably due to extensive use of assisted reproductive technologies and increased pelvic infections.

A total of 36 ectopic pregnancies were managed from April 2007- March 2008 among which 33 were ruptured ectopics with haemoperitoneum, treated surgically by emergency laparotomy and salpingectomy. Three unruptured cases were treated with single dose of intramuscular methotrexate followed by serum $\beta$ hCG measurement.

Transvaginal sonography reassured that medical management of unruptured ectopic pregnancies with systemic single dose of methotrexate was successful with few side effects and a cost effective alternative to traditional surgical management for unruptured ectopic pregnancies.
\end{abstract}

Keywords: Single dose methotrexate, unruptured ectopic pregnancy

\section{INTRODUCTION}

Ectopic pregnancy (EP) defined as gestation outside the normal uterine cavity continues to be one of the most common gynecologic emergencies, leading to multiple morbidities and mortalities resulting in about $9-13 \%$ of death. ${ }^{1}$ Most of the EP( ruptured and un-ruptured) are diagnosed much earlier due to sensitive ß-human chorionic gonadotropin (BhCG) assays and transvaginal ultrasonography (TVS).Consequently a prompt diagnosis followed by quick resuscitation and surgical interventions (laparotomy with either salpingectomy, salpingostomy or salpingotomy) continues to be the main stay of treatment worldwide particularly in an undeveloped country like Nepal.

\section{CORRESPONDENCE}

Dr. Suniti Rawal

Lecturer,Department of Obstetrics and Gynecology

Tribhuvan University Teaching Hospital,Kathmandu, Nepal

Phone: 9851002183

Email: sunudinurawal@yahoo.com
Laparoscopic surgeries for both unruptured and stable ruptured EPs are emerging as alternative treatment option in some of the centers of Nepal too. However given that laparoscopy is not widely available in Nepal and with relatively less expertise in this field, medical treatment with methotrexate for the unruptured EP seems to be an attractive option.

Hreschchyhyn et al, in 1965 was the first to report the use of methotrexate in the management of EP followed by Tanaka et al. ${ }^{2}$ treatment of an interstitial EP with a 15-days course of intramuscular methotrexate in 1982. Accordingly methotrexate therapy in a single intramuscular (IM) dose of $50 \mathrm{mg} / \mathrm{m}^{2}$ has been reported to be the widely used and accepted medical management to resolve EP in about $94 \%$ of the treated population with very negligible side effects. ${ }^{3}$ 
Rawal. Successful use of Single dose of Methotrexate for the treatment of Unruptured Ectopic Pregnancy at Tribhuvan University.

Nevertheless the main reason for restricted use of methotrexate in treatment of EP could apparently be the fear of tubal rupture and the complications that may arise thereafter.

Methotrexate, a folic acid analog that interferes with DNA synthesis competitively binds the enzyme dihydrofolic acid reductase which converts dihydrofolate to tetrahydrofolate, thus prevents the reduction of folate to its active form, tetrahydrofolate. Without tetrahydrofolate, DNA synthesis, repair and cellular replications are impaired. It is ideal for inhibition of rapidly growing cells such as trophoblasts. ${ }^{4}$

Since methotrexate is a drug that has many side effects, its dose and the duration should be considered with utmost carefulness. Side effects like severe leukopenia, thrombocytopenia, bone marrow aplasia, ulcerative stomatitis, diarrhea, liver cell necrosis are frequently observed in patients receiving methotrexate for a prolong time in contrast to single-dose administration that has negligible side effects. ${ }^{5}$

In these case series, three cases managed successfully with a single dose of methotrexate are reviewed with reference that the correct choice of cases may have a favorable outcome with medical management.

\section{CASE SERIES}

In the Department of Obstetrics and Gynecology, female surgical ward in a span of 1 year from April 2007-March 2008, total 36 cases of EP were managed, out of which 33 ruptured EP with haemoperitoneum were managed surgically by emergency laparotomy and salpingectomy and 3 unruptured EP were treated with a single dose of IM methotrexate.

All these cases included a history of overdue periods with positive urine pregnancy test, normal vital parameter at the time of presentation with transvaginal scan (TVS) showing empty uterine cavity, presence of an adenexal mass and had no evidence of haemoperitoneum. Therefore all the unruptured EPs diagnosed with serum $\beta$ hCG $<10,000$ $\mathrm{mIU} / \mathrm{ml}$, tubal pregnancy diameter $<3 \mathrm{~cm}$ by TVS, absence of hemoperitoneum and absence of fetal cardiac activity in USG were considered for the medical management with methotrexate.

Post briefing the patient and the family members about the drug, its side effects and the possible need for emergency laparotomy, the therapy started with the regime of $1 \mathrm{mg} /$ $\mathrm{kg}$ body weight or $50 \mathrm{mg} / \mathrm{m}^{2}$ body surface area(BSA), calculated from a nomogram with the use of height and body weight. As a secondary measure, a repeat dose of methotrexate was planned if the serum $\beta$ hCG level did not fall $\geq 15 \%$ and below on the 4 th and 16 th day respectively from the initial day.
Thirty five years $\mathrm{P}_{1+1}$ visited a nearby clinic for overdue of menstrual periods by 2 weeks followed by pain abdomen for past 10 days along with irregular periods for past 5 months. As she was not sure of her LMP, urine pregnancy test was advised with result coming out as positive, followed by TVS which showed left sided adnexal mass of $2.3 \mathrm{~cm}$ with an empty uterine cavity and absence of any collection either in the pelvic or the abdominal cavity. She was referred to TUTH for further management with the diagnosis of EP.

On admission, the patient was found to be haemodynamically stable, with soft abdomen and no guarding or rigidity. Per speculum examination showed normal cervix, per vaginally the uterus was anteverted, normal size, mobile and the fornices were clear. She was planned to be treated medically following the report of serum ßhCG, which was $1700 \mathrm{mlU} / \mathrm{ml}$.

With consent from the patient and her family all the preliminary investigations like the haemoglobin and PCV complete blood count (CBC), liver function test (LFT), renal function test (RFT) and blood grouping and Rhesus typing were performed and methotrexate was given. Methotrexate in the dose of $1 \mathrm{mg} / \mathrm{Kg}$ body weight, which was $50 \mathrm{mg}$, was given intramuscularly, considering the weight of the patient $(49 \mathrm{~kg})$. She had complained of slight epigastric pain that was relieved by the $\mathrm{H}_{2}$ blockers.

On the 4th day serum $\beta$ hCG was repeated, the leve declined to $316 \mathrm{mIU} / \mathrm{ml}$ and similarly on the 16th and the 30th day the serum $\beta$ hCG levels went down to $20 \mathrm{mlu} /$ $\mathrm{ml}$ and $0.5 \mathrm{mIU} / \mathrm{ml}$ respectively. Serial TVS done on the $4^{\text {th }}, 7^{\text {th }}$ and $16^{\text {th }}$ day showed the diameter of the adnexal mass reduced to $1.5 \mathrm{~cm}, 7 \mathrm{~mm}$ and $2 \mathrm{~mm}$ respectively and it completely resolved on the $30^{\text {th }}$ day of administration of methotrexate. She was discharged on the 11th day and was advised to follow up at OPD.

\section{Pretreatment TVS}

Transvaginal sonography
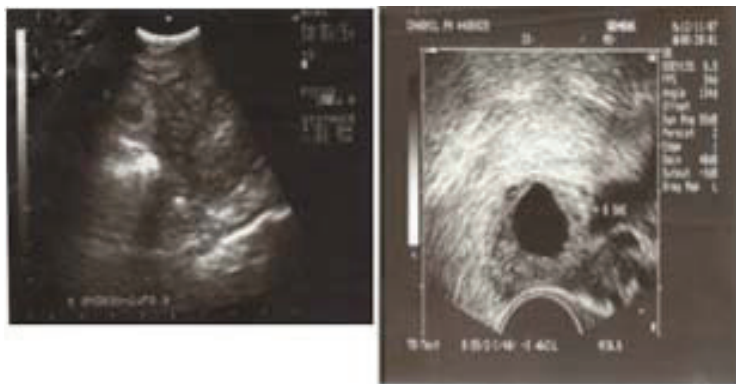

Fig 1. Pre methotrexate TVS-Adenexal mass1.9x1.6

\section{CASE 1}



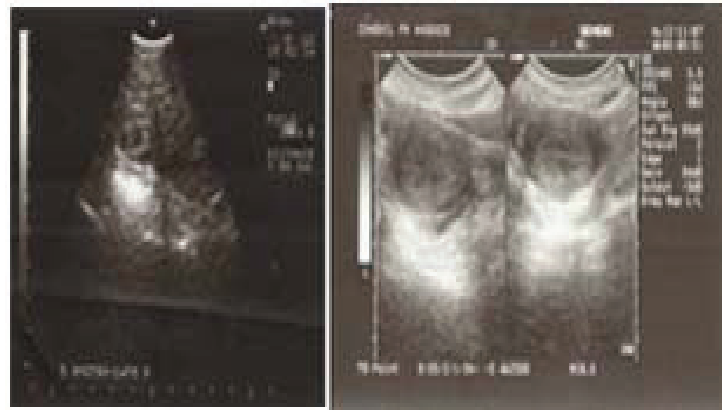

Fig 2. Post Methotrexate GS $0.8 \mathrm{~cm}$

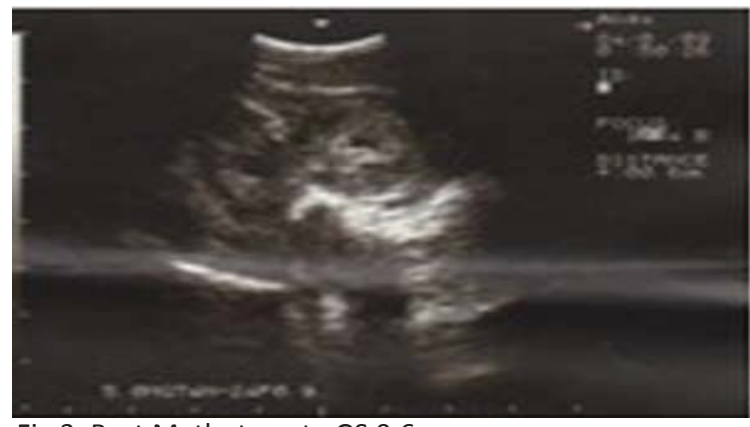

Fig 3. Post Methoterxate GS $0.6 \mathrm{~cm}$

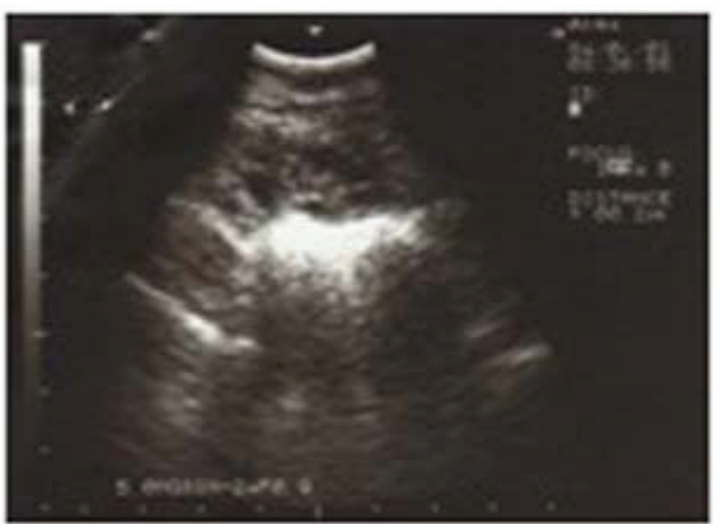

Fig 4. Post Methotrexate GS $0.2 \mathrm{~cm}$

\section{CASE 2}

Twenty seven years nullipara presented to emergency (ER) with the complaints of overdue by 11 days ( 5 weeks and 4 days gestation) associated with pain abdomen and per vaginal bleeding for 3 days.

She was on oral contraceptive pills and had discontinued recently. Urine pregnancy test was positive. On examination she was found to be haemodynamically stable with soft non tender abdomen. On per speculum examination, there was slight blood stained discharge and per vaginally, the uterus was anteverted, normal size, mobile and fornices clear. Cervical motion tenderness was absent. TVS revealed a small hyper echoic lesion in the right adnexa with a small cystic lesion within measuring $1.9 \times 1.7 \mathrm{~cm}$. The uterine cavity was empty and the endometrial lining was $4 \mathrm{~mm}$.
Serum b hCG was $1665 \mathrm{mlU} / \mathrm{ml}$ on the day of admission. The baseline investigations like blood grouping and Rhesus typing, haemoglobin, PCV, CBC, LFT, RFT were normal. She weighed $51 \mathrm{~kg}$, thus the dose of methotrexate calculated from $1 \mathrm{mg} / \mathrm{Kg}$ body weight was $50 \mathrm{mg}$ given IM.

The gradual decline of serum $\mathrm{b}$ hCG to $220 \mathrm{mIU} / \mathrm{ml}$ was noted on the $4^{\text {th }}$ day, $12 \mathrm{mIU} / \mathrm{ml}$ on the $16^{\text {th }}$ day and $0.7 \mathrm{mlU} / \mathrm{ml}$ on $29^{\text {th }}$ day. Similarly TVS done showed the right adenexal mass with a cyst gradually decreasing to $1.2 \mathrm{~cm}, 8 \mathrm{~mm}$ and $6 \mathrm{~mm}$ on the $4^{\text {th }}, 6^{\text {th }}$, and $16^{\text {th }}$ day post methotrexate and completely resolved on the $29^{\text {th }}$ day. She was discharged on the $6^{\text {th }}$ day of admission with regular visits to GOPD with report of serum b hCG and TVS.

\section{Pretreatment TVS}
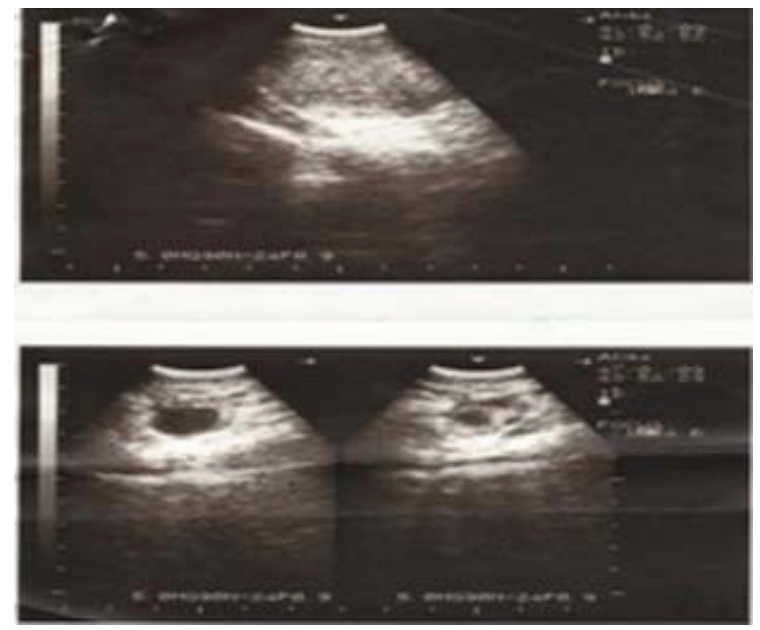

\section{CASE 3}

Forty one years $\mathrm{P}_{3+1}$ at 8 weeks +4 days amenorrhea visited a nearby clinic for the positive urine pregnancy test performed at home. As she had a history of irregular cycles for the past 2-3 months, urine pregnancy test and TVS were repeated at the clinic that showed uterus anteverted, normal size with normal echo texture and endometrial lining, an empty uterine cavity and gestational sac in the right adnexae with minimal free fluid in the Pouch of Douglas (POD).

She was referred to a sonologist for an expert opinion where repeat USG revealed very thick endometrium of $23 \mathrm{~mm}$ with no intrauterine pregnancy, 2 nodules in the left adnexal region, $1 \mathrm{st}$ ?corpus luteum $20 \times 15 \mathrm{~mm}$ and the $2 n d$ ? $8 \times 7 \mathrm{~mm}$. With the diagnosis of EP she was referred to TUTH for better management.

On examination she was haemodynamically stable. Abdomen was soft and non tender. Per speculum examination revealed a normal cervix and per vaginally the uterus was anteverted, normal size with clear fornices. There was no cervical motion tenderness. 
Patient was admitted and was planned for medical management after the serum b hCG report. She and her husband were counselled for both the medical management as well as any need for possible emergency laparotomy.

The preliminary examination as in the earlier cases was done. Serum b hCG was $9100 \mathrm{mlU} / \mathrm{ml}$. The patient weighed $65 \mathrm{Kg}$ and the height was $156 \mathrm{~cm}$, and the dose of methotrexate calculated by $50 \mathrm{mg} / \mathrm{m}^{2}$ BSA came to 1.67 $\mathrm{m}^{2}$ that was $80 \mathrm{mg}$ administered IM. Serum b hCG done on day $5,12,19,26,30$ and 35 were 2030, 680, 236, 127, 40 and $15 \mathrm{mIU} / \mathrm{ml}$ respectively. TVS was done twice weekly to observe the decrease in the size of the mass as well as to rule out haemoperitoneum. She was discharged on the 6th day of admission and was followed up at GOPD with the serum b hCG report and TVS.

None of these 3 cases required second dose of methotrexate or emergency laparotomy.

\section{DISCUSSION}

Ectopic pregnancy is a high-risk condition that occurs in $1.9 \%$ of reported pregnancies ${ }^{6}$ and still remains one of the leading causes of maternal morbidity as well as mortality. Due to the advances in the assisted reproductive techniques the incidence of the EP is recently at its highest.

Surgical management of EP is the mainstay of treatment, be it in the open form or in a minimally assisted ways as laparoscopic surgeries.

Memorial medical treatment for unruptured EPs has also catered to provide patient with simplified, non surgical and cost effective way of management, though the fear of rupture always looms large during such therapies. Medical management offers success rate comparable to surgical management, with no loss in future fertility.

Single dose methotrexate is more commonly used owing to its convenience, simplicity and lesser side effects. Methotrexate can be given in a regimen of $1 \mathrm{mg} / \mathrm{kg}$ body weight or $50 \mathrm{mg} / \mathrm{m}^{2} \mathrm{BSA}$, given in the 3 cases with good results. The overall success rate for women treated with methotrexate using either regimen was $89 \%$ (1181 of1327) in a review conducted by Barnhart et al. ${ }^{4}$

All 3 patients presented to us were lucky enough not to require surgeries, as all of them were haemodynamically stable and could come to us when intervention was most needed. They were of different age groups and parities. All of them had overdue periods with urine pregnancy test positive, though without the help of TVS the intra or extra uterine pregnancy could not have been possibly ruled out.

Prompt TVS evaluation done in all 3 cases was the key factor in diagnosing the unruptured EP that showed adnexal mass with an empty uterine cavity. The TVS results combined with the serum b hCG level usually helps in confirming the EPs too. A study performed by Lozeau and Potter ${ }^{6}$ reported that if serum $\beta$ hCG level is $\geq 1,500 \mathrm{mlU} / \mathrm{ml}$ with TVS showing no intrauterine gestational sac, ectopic pregnancy should be suspected which was consistent in all 3 cases that had serum $\beta$ hCG levels of 1700,1665 and $9100 \mathrm{mIU} / \mathrm{ml}$ with no intrauterine pregnancy in TVS.

The serum $\beta$ hCG done at the time of admission varied probably due to different gestational ages at the time of presentation, though all were below $10,000 \mathrm{mIU} / \mathrm{ml}$. Studies have shown that, the lower the level of serum $\beta$ hCG the higher is the chance of successful treatment with single dose methotrexate. A study conducted by Catherine ${ }^{7}$ showed that no women with pretreatment serum $\beta$ hCG $\geq 5000 \mathrm{mlU} / \mathrm{ml}$ successfully responded to single dose of methotrexate, thus stating that the single dose regimen appeared to correlate with the absolute value of serum $\beta$ hCG level, as defined by the trophoblast mass and not the rate or direction of pretreatment serum $\beta$ hCG change. Only 1 case had a serum $\beta$ hCG level $\geq 9000 \mathrm{mlU} / \mathrm{ml}$, but did respond well to the single regimen. Similarly both Mol et al ${ }^{8}$ and Sowter ${ }^{9}$ have concluded that laparoscopic surgery is the most cost effective treatment for tubal EP and systemic methotrexate is a good alternative in selected patients with mild symptoms and low serum $\beta$ hCG concentration.

Various studies have shown that the success rate of treatment between the single and the multiple dose regimen were comparable and that the multiple doses of methotrexate was less effective than a single dose ${ }^{10,11}$ though none of the patients in this study required a second dose. Decrease in the serum $\beta$ hCG was observed on the 4 th, 16th and around the 29th day. As expected, following the 1st dose of methotrexate, the decrease in the level of serum $\beta$ hCG was $\geq 15 \%$ to that of the initial serum $\beta$ hCG, which did occurred in all the 3 cases. Due to the cost constrains, serum $\beta$ hCG was not done as frequently as stated in the books and literatures where an additional dose of methotrexate was suggested if the serum $\beta$ hCG levels were above the value of the curve on day 2, 4, 7 or 10 and 12.

TVS was done in all the cases for monitoring as well as for the follow up and decrease in the size of adnexal mass was marked as successful treatment. Performing TVS routinely is not usually recommended, but is a preferred tool in cases where cardiac activity is still present, and is recommended till the cardiac activity disappears. But probably performing TVS in all these cases was worthwhile to see both the decrease in the size of adenexal mass as well as to detect haemoperitoneum if any.

The resumption rate is defined as time interval between the initiation of therapy and the undetectable serum $\beta$ hCG. In all the 3 cases the resumption rate was 28-35 days. Consistent to findings of our study is that done in Northwestern Memorial hospital, where the mean time for 
complete resolution was $23.3 \pm 13$ days in patients treated with single dose regimen. ${ }^{7}$ Contrary to this, study by Slaughter ${ }^{5}$ has shown the resolution time to be as longer as 70-120 days. The longer the unruptured pregnancy takes to resolve the higher is its chance to get ruptured. The longest time from initial treatment to rupture has been reported to occur at 31 days and that too after 30 days of treatment.

Very little side effects were noted as in the 1st case where epigastric pain occurred. Much severe side effects have been seen with the use of multiple rather than the single dose. $^{11}$

Hospital stay varied from 6-11days.The 1st case was admitted for a longer time as compared to 2 nd and $3^{\text {rd }}$. The cost factors for medical management was comparable to that of laparotomy. Even after adding up all the cost for medical management, it would be comparatively a bit

\section{REFERENCES}

1. Fylstra D. Tubal pregnancy: A review of current diagnosis and treatment. Obstet Gynecol. 1998;53(5):320-7.

2. Tanaka T, Hayashi H, Fujimoto S, Ichinoe K. Treatment of interstitial ectopic pregnancy with methotrexate: report of a successful case. Fertil Steril. 1982;37(6):851-2.

3. Stovall TG, Ling FW, Buster JE. Single dose methotrexate: an expanded clinical trial. Am J Obstet Gynecol. 1993;168:1759-65.

4. Barnhart K T, Gosman G, Ashby R, Sammel M. The medical management of Ectopic Pregnancy: A meta-analysis comparin single dose and multidose regimens. Am J Obstet Gynecol. 2003;101(4):778-84

5. Slaughter JL, Grimes DA: Methotrexate therapy - Nonsurgical management of ectopic pregnancy. West J Med. 1995;162(3):225-8.

6. Lozeau AM, Potter B. Diagnosis and management of ectopic pregnancy. Am Fam Physician. 2005;172(9):1707-14.

7. Catherine S S, Anderson L, Marilynn CF. Single dose Methotrexate for the treatment of ectopic pregnancy: Northwestern Memorial Hospital three-year experience. Am J Obstet Gynecol. 1996; 174(6):1840-8. cheaper than needed for laparotomy.

Laparoscopic surgeries have taken an upper hand in the treatment of EP, and it is considered to be superior to medical treatments in various studies too. ${ }^{10,11}$ But due to the unavailability of laparoscopic expertise round the clock this has not been feasible in our hospital.

\section{CONCLUSION}

In country like ours it seems worthwhile trying the medical management provided the patient arrives at the earliest, are haemodynamically stable and are willing to come for follow up.

With strict criteria of inclusion and follow up, single dose IM methotrexate is a successful method for treatment in selected cases of unruptured ectopic pregnancy, with very few side effects.

8. Mol F, Mol BW, Ankum WM, van der Veen F, Hajenius PJ. Current evidence on surgery, systemic methotrexate and expectant management in the treatment of tubal ectopic pregnancy: a systematic review and Meta analysis. Hum Reprod Update. 2008;14(4):309-19.

9. Sowter MC, Farquhar CM, Petrie KJ, Gudex G. A randomized trial comparing single dose systemic methotrexate and laparoscopic surgery for the treatment of unruptured tubal pregnancy. BJOG. 2001;108(2):192-203

10. Guvendag GES, Dilbaz S, Dilbaz B, Aykan Yildirim B, Akdag D, Haberal A. Comparison of single and multiple dose methotrexate therapy for unruptured tubal ectopic pregnancy: a prospective randomized study. Acta Obstet Gynecol Scand. 2010;89(7):889-95.

11. Lipscomb GH, Givens VM, Norman ML. Comparison of single and multiple dose methotrexate protocol for the treatment of ectopic pregnancy. Am J Obstet Gynecol. 2005;192(6):1844-8. 